\title{
Doing two things at once: The role of temporal compatibility
}

\author{
STUART T. KLAPP \\ California State University, Hayward, California 94542
}

\begin{abstract}
Performance in a periodically repeating keypress response was measured as a function of the relation between the response patterns required of the two hands. Compared to identical left- and right-hand responses, performance was degraded when the temporal periods of the left- and right-hand responses were not harmonically related. By contrast, performance was not degraded compared to the identical task control when the left- and right-hand responses had the same or harmonically related periods. These findings suggest a limitation in parallel generation of multiple time frames that is assumed to be associated with a late stage of central processing in which response commands are generated.
\end{abstract}

Can people carry out two tasks simultaneously without mutual interference? One tradition regarding this issue holds that we cannot, that is, that processing is limited to a single channel (e.g., Craik, 1948; Vince, 1948). In this view, if a second stimulus arrives while processing of a stimulus is still in progress, processing of the second stimulus must be delayed until processing of the first stimulus has been completed. This leads to the "psychological refractory period" effect (Herman \& Kantowitz, 1970; Welford, 1968). By contrast, other approaches emphasize the possibility that, under appropriate conditions, multiple processes can occur in parallel (Greenwald \& Shulman, 1973; Schneider \& Shiffrin, 1977; Shiffrin \& Schneider, 1977). Thus the single-channel limit may not apply universally.

Other issues concern the locus within the flow of information of any limit in dual processing. Does this restriction occur prior to or after extraction of meaning from the stimulus (e.g., Lewis, 1970; Treisman, Squire, \& Green, 1974)? Or is there a "floating" restriction due to limited processing capacity that can be allocated to various stages of processing (e.g., Johnston \& Heinz, 1978, 1979; Kahneman, 1973; Moray, 1967)?

This research was supported by Grant BNS 76-13466 from the National Science Foundation. George Eggleton and Doug Filangeri assisted with the equipment. Pearl Barker, Carol Daniels, Mark Hagerty, and Victor Pereira assisted with Experiment 1 and with pilot experiments. Robert Snider carried out Experiment 2. Helene Brodrick, Sabine Dickerson, and Margie Gasper carried out Experiments 3 and 4 and assisted with manuscript preparation. Experiment 3 was reported by Brodrick, Dickerson, and Gasper at the Western Psychological Conference for Undergraduate Research, Santa Clara, California, May 1979. After Experiments 1 and 2 were completed, the author became aware (August 1978) of rather similar work designed and being initiated by Diana Deutsch. The author expresses appreciation for useful discussions with Dr. Deutsch at that time. Requests for reprints should be sent to Stuart T. Klapp, Department of Psychology, California State University, Hayward, California 94542.
The previous research on single-channel limits focused on limitations encountered during stimulus encoding, decision making, and other relatively early processes. By contrast, the present analysis examines limitations that may arise in the system that organizes or programs responses. The notion to be explored holds that this system is limited in producing more than one simultaneous temporal pattern or rhythm. Thus, although it should be relatively easy to generate simultaneous responses with different muscles if the responses follow the same temporal pattern, even very simple sets of responses that cannot be accommodated to the same time frame should produce marked mutual interference. This notion adds yet another dimension to the issue of dual-task limitations.

The experiments reported here tested a more specific formulation of this general notion. Two periodically repeating response sequences were defined as temporally compatible (i.e., operating on the same time frame) if and only if their periodic repetitions are in harmonic relation. This definition might be extended to nonrepeating responses by assuming that each response represents one period of what could be extended to a periodically repeating sequence. The principle tested holds that two temporally compatible responses can be generated in parallel with little or no interference even if the responses are complex. By contrast, even relatively simple responses that are temporally incompatible will interfere with each other. The present experiments tested the applicability of this formulation to a simple situation in which two telegraph keys were manipulated by the two hands using the same rhythm, harmonically related rhythms, or nonharmonic rhy thms.

\section{EXPERIMENT 1}

\section{Method \\ Conditions and task. Two standard telegraph keys located on a table and spaced $68 \mathrm{~cm}$ apart to the subject's right and left}


were manipulated by the right and left hands. A light stimulus was centered between the telegraph keys. The experiment was a 4 by 3 factorial design with four task conditions at three stimulus rates.

In the "Thythm" condition, the light signal indicated the temporal pattern at which a telegraph key was to be pressed with the left hand. The light was on for $t \mathrm{sec}$ and then off for $t \mathrm{sec}$, where $t$ was $.3, .6$, or $1.2 \mathrm{sec}$, defining the stimulus rate variable. The instructions to the subject were to position the telegraph key down when the light was on and up when the light was off. The performance measure was the mean proportion of time of a $20-\mathrm{sec}$ scored interval during which the response and stimulus did not agree (key up while light on or key down while light off). The right hand was idle in the rhythm condition.

In the "press fast" condition, the left hand was idle. The right hand pressed the telegraph key as fast as possible, and the mean number of presses during the 20 -sec trials was the performance measure. The light stimulus was active, but its rate should not influence performance since no response was to be linked to this stimulus.

In the "Ihythm plus press fast" condition, the two tasks described above were done together. The assumption underlying this condition was that, if the instruction to press as quickly as possible with the right hand were followed, then the right-hand response would have a timing pattern that would not be harmonically related to that commanded by the signal for the left hand. Therefore, interference would be observed relative to the above single-task conditions.

In the "identical" condition, the subject pressed the right and left keys at the same rate, as determined by the light stimulus. Left-hand performance should be equivalent to that in the single-task control (rhythm condition), according to the notions being tested.

For the rhythm, rhythm plus press fast, and identical conditions, left-hand performance was the primary measure, even though all subjects were right-handed. This arrangement was selected since pilot work showed that some subjects were unable to press the key very quickly with the left hand, but they could do so with the right hand. Therefore, the fast-pressing task was assigned to the right hand, and the primary task of rhythm keeping was assigned to the left hand.

Design. Each of the eight subjects received all four task conditions at all three stimulus rates. The order in which the task conditions appeared was balanced across subjects as determined by two Latin squares. The sets of subjects assigned to each Latin square received the stimulus rates in opposite order, ascending for one set of subjects and descending for the other set. All of the rates $(.3, .6$, and $1.2 \mathrm{sec})$ for a given task were presented before moving on to a new task. The experiment comprised two daily sessions, with the entire design replicated in the same sequence during each session. Only data from the second session were analyzed.

Each cell in the design was represented as three trials, each of which involved a 20 -sec period of performance measurement. Only the last two trials were scored. On each trial the stimulus

Table 1

Proportion of Time During Which Stimulus and Response Did Not Correspond for Left Hand in Experiment 1

\begin{tabular}{lcccc}
\hline & \multicolumn{4}{c}{$\begin{array}{c}\text { Left Stimulus Rate } \\
\text { (Seconds On and Off) }\end{array}$} \\
\cline { 2 - 6 } Condition & $.3 / .3$ & $.6 / .6$ & $1.2 / 1.2$ & Mean \\
\hline Rhythm (Right Idle) & .26 & .12 & .11 & .16 \\
Identical & .30 & .13 & .11 & .18 \\
Rhythm Plus Press Fast & .40 & .24 & .13 & .26 \\
Mean & .32 & .16 & .12 & \\
\hline
\end{tabular}

Table 2

Mean Number of Presses per 20-Sec Trial with Right Hand in Experiment 1

\begin{tabular}{lrrrr}
\hline & \multicolumn{4}{c}{$\begin{array}{c}\text { Left Stimulus Rate } \\
\text { (Seconds On and Off) }\end{array}$} \\
\cline { 2 - 5 } \multicolumn{1}{c}{ Condition } & $.3 / .3$ & $.6 / .6$ & $1.2 / 1.2$ & Mean \\
\hline Press Fast (Left Idle) & 126 & 126 & 126 & 127 \\
Rhythm Plus Press Fast & 93 & 115 & 118 & 109 \\
Mean & 110 & 121 & 123 & \\
\hline
\end{tabular}

was activated for about $10 \mathrm{sec}$ prior to taking measurements, and then the stimulus remained activated for an additional 5 to $10 \mathrm{sec}$ after measurements were completed. Thus the 20 -sec period of measurement avoided the start-up or wind-down effects at the beginning or end of the period of performance. A rest interval of approximately $10 \mathrm{sec}$ separated the trials.

Subjects. The eight right-handed subjects were students at California State University, Hayward, who participated as part of one option of a course requirement or for pay.

\section{Results and Discussion}

Left hand. Table 1 displays the mean proportion of the time during the 20 -sec scored intervals for which the left-hand response did not correspond to the stimulus (i.e., key down but light off or key up but light on). Performance was less accurate at the faster rates $[F(2,14)=13.5, p<.001]$ and differed among the three task conditions for which the left hand responded $[\mathrm{F}(2,14)=20.2, \mathrm{p}<.001]$. The timing and task variables tended to interact $[F(4,28)=2.6, .05<p<.10]$, but this apparent interaction did not involve a reversal of any of the effects.

For the rhythm plus press fast condition, left-hand performance was poor compared with the single-rhythm control $[F(1,7)=43.0, p<.001]$. In contrast to this significant degradation in performance when the tasks were of incompatible timing, requiring the right hand to follow the left-hand rhythm in the identical condition did not significantly reduce left-hand performance compared with the single-task control $[\mathrm{F}(1,7)=1.7$, $\mathrm{p}>.2]$. These results support the notion that two tasks will interfere more if they are temporally incompatible.

Right hand. If the instruction to press as rapidly as possible was strictly followed for the press fast and rhythm plus press fast conditions, the rate of right-hand pressing would be the same in the two conditions. However, as Table 2 indicates, there was a tendency for fewer presses to occur in the combined task $[F(1,7)=5.31, .05<p<.10]$, suggesting the possibility that the presence of a concurrent incompatible left-hand task may produce interference with the right-hand task even though the instructions were to maximize righthand performance in both conditions.

\section{EXPERIMENT 2}

In Experiment 1 left-hand performance was poorer when the right hand was performing the press fast task 
than when it was performing the identical task. The theoretical notions under consideration attribute this finding to a lack of temporal compatibility between the left- and right-hand tasks in the press fast condition. However, an alternative interpretation would be that the increased amount of right-hand pressing in the press fast condition relative to that in the identical condition control is responsible for this result. Experiment 2 was designed, in part, to test this alternative interpretation. A temporally incompatible condition (unsynchronized) was included, for which the right hand made fewer responses than in the identical response control. According to the notion of temporal compatibility, but not according to the alternative view concerning response rate, left-hand performance should be markedly worse in this (unsynchronized) condition than in the identical response condition.

An alternative interpretation of a different aspect of the results of Experiment 1 would be that the ease of doing two responses at once is restricted to the case in which the left- and right-hand responses are identical. In Experiment 2 the identical response condition was compared with a condition (skip) in which the response patterns involved different but harmonically related periods. According to the viewpoint under test, performance in this "skip" condition should be as good as that in the identical response condition, and markedly better than that in the unsynchronized or press fast condition.

Finally, it is not clear from Experiment 1 how practice might influence the response decrement associated with temporal incompatibility. It is possible that, with more practice, the decrement associated with temporal incompatibility might vanish. Experiment 2 examined this possibility by testing over 10 sessions of practice.

\section{Method}

Overview. Each subject received 10 sessions of approximately $30 \mathrm{~min}$ each, with all four conditions represented in each session. These conditions differed with respect to the task required of the right hand. The left-hand task for all conditions was to press and release the telegraph key for a cycle of $.6 \mathrm{sec}$ down and $.6 \mathrm{sec}$ up, as defined by a $2,900-\mathrm{Hz}$ tone in the left ear. When the tone was on, the key was to be down, and when the tone was off, the key was to be up. Left-hand performance was measured as the mean proportion of time during the 20 -sec trials in which the press response did not correspond to the tone stimulus (key up but tone on or key down but tone off). A similar measure of right-hand performance was taken during the conditions for which a comparison would be possible.

Conditions. Each condition was described to the subject before any trials were run, since one object of the experiment was to investigate improvement with practice rather than the rate of intellectual discovery of the nature of the conditions. In the "identical" condition, the right hand was to make exactly the same response as the left hand and at the same timing (.6 sec key down, $.6 \mathrm{sec}$ key up). A $4,500-\mathrm{Hz}$ tone was presented to the right ear in phase with the one to the left ear. In the "skip" condition, the tone to the right ear for the right-hand response directed a $.6-\mathrm{sec}$ press (as in the identical condition), but the tone and the corresponding press occurred only every second cycle rather than on every cycle. Thus there was a 1.8 -sec interval between .6 -sec tones and presses, yielding a total period of $2.4 \mathrm{sec}$. Although the periods of repetition were different for the left-and right-hand responses in this condition, they were in harmonic relation (i.e., $1.2 \mathrm{sec}$ for left and $2.4 \mathrm{sec}$ for right). Therefore, according to the view being tested, performance should be equally good in the skip as in the identical condition, even though the left and right hands made very different response sequences in the skip condition and the same responses in the identical condition.

The remaining conditions are designated as not compatible, since the periods of repetition for the left and right hands were not in harmonic relation. In the "unsynchronized" condition, the tones provided for the right hand, and the corresponding press responses required, were similar to those in the skip condition except for the exact timing (and the relation of timing between the hands). For four of the eight subjects, the timing was $2.0 \mathrm{sec}$ off and $.6 \mathrm{sec}$ on, yielding a period of $2.6 \mathrm{sec}$, which is not harmonically related to the 1.2-sec period for the left-hand tone and response. For the remaining subjects, the timing was $1.6 \mathrm{sec}$ off and $.6 \mathrm{sec}$ on, producing a period of $2.2 \mathrm{sec}$, which again is not harmonically related to the $1.2-\mathrm{sec}$ period of the left-hand tone and response. Even though the rate of responding of the right hand was less in the unsynchronized condition than in the identical condition, the theory predicts more interference in the unsynchronized condition.

In the "press fast" condition, the subject was to press with his right hand as quickly as possible. No tone signal was provided for the right ear.

Design. Each trial was composed of the same sequence of events as in Experiment 1. Each condition was presented as a consecutive set of eight trials, with data recorded from the final six trials of the set. The order in which the four conditions were presented remained constant from session to session for a given subject, but the order was balanced across subjects.

Subjects. The eight right-handed subjects were from the same population as in the previous experiment. Two subjects were replaced without reporting their data. In one case the experiment was terminated because performance was below $50 \%$ during the first two sessions, and in the other case, the subject quit attending the experiment and could not be induced to continue.

\section{Results and Discussion}

Left hand. Left-hand performance (Figure 1) differed among the conditions of concurrent right-hand tasks

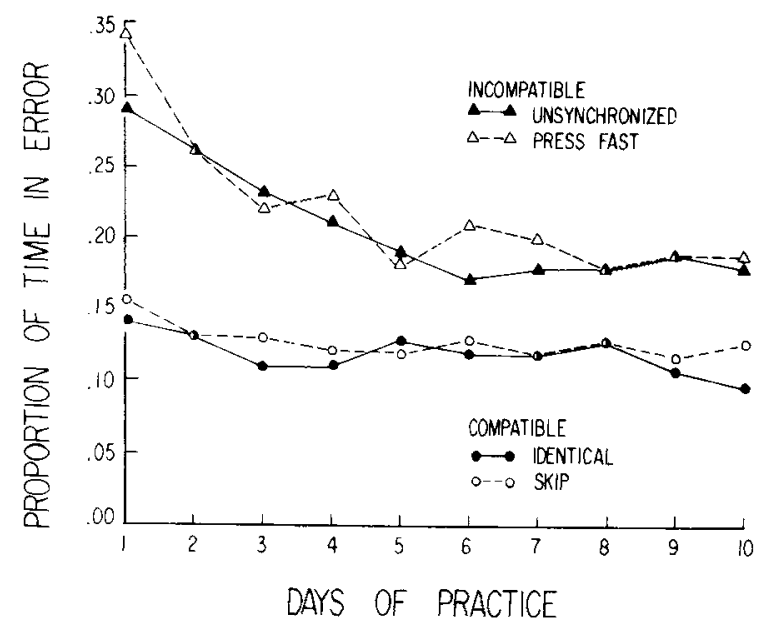

Figure 1. Left-hand performance as a function of concurrent right-hand task and days of practice in Experiment 2. 
$[F(3,21)=11.07, p<.001]$ and improved with days of practice $[F(9,63)=6.44, p<.001]$. The condition and practice variables interacted $[\mathrm{F}(27,189)=2.84$, $\mathrm{p}<.001]$. The following paragraphs provide further analysis of these overall effects.

Left-hand performance did not differ between the two temporally compatible conditions, identical and skip $[F(1,7)<1]$, and there was no interaction of any difference between these conditions by practice $[F(9,63)<1]$. Equivalent performance in these conditions was expected because the left- and right-hand responses had compatible timing in each condition. However, it should be noted that the conditions differed greatly with respect to the degree of similarity of the right- and left-hand responses. Left- and right-hand responses were identical in one condition and different in the other. Apparently, two different responses that have harmonically related periods can be generated as easily as two identical responses.

Left-hand performance did not differ between the two incompatible conditions, unsynchronized ${ }^{1}$ and press fast $[F(1,7)<1]$. The theory makes no prediction about the relation between performance in these conditions, but it does predict that left-hand performance should be markedly worse in these conditions compared with the compatible conditions. Consistent with this prediction, each of the four possible comparisons between compatible and incompatible conditions was significant [with at least $F(1,7)=11.6, p<.025$ ] In particular, the identical condition led to markedly better performance than the unsynchronized condition $[F(1,7)=38.58, p<.001]$, even though the right-hand task involved a larger number of responses in the former case. These data strongly suggest that temporal compatibility rather than the rate of responding determines the amount of interference.

In general, performance improved over the first 5 days of practice $[F(4,28)=8.89, p<.001]$, but not over the final 5 days $[F(4,28)<1]$, suggesting that the experiment was carried out long enough for performance to reach a stable level. During the first 5 days, the difference among conditions interacted with practice such that performance improved most rapidly for the incompatible conditions $[\mathrm{F}(12,84)=2.78, \mathrm{p}<.005]$. If this interaction had continued over 10 days, one might expect that, with even more practice, the interference due to lack of temporal compatibility might disappear. However, this interaction did not appear during the last 5 days of practice $[F(12,84)<1]$, and the four conditions differed with respect to left-hand performance during the last 5 days after performance was stabilized $[F(3,21)=6.07, p<.005]$. Therefore, it appears that there is a large component of interference between temporally incompatible responses that cannot be overcome with practice.

Right hand. For the right hand, it was possible to compare performance in the skip and unsynchronized conditions because, on the average, the rate of required right-hand responding was equivalent. As is apparent in Table 3, right-hand performance was worse for the incompatible unsynchronized condition than for the compatible skip condition at all levels of practice $[F(1,7)=36.94, p<.001]$. Thus the interference involving temporally incompatible responses is mutual; that is, both left and right responses exhibit the interference.

\section{EXPERIMENT 3}

One possible problem with the unsynchronized condition of Experiment 2 is the possibility that the tone presented to one ear might have interfered with perception of the tone presented to the other ear, even though the two tones differed in frequency. Therefore, perceptual interference rather than incompatibility of response timing might have produced the decrement of performance for the unsynchronized condition relative to the compatible conditions. As a test of this perceptual interference interpretation, Experiment 3 involved a comparison of performance in the dual-tone procedure (tone-tone) with performance when a tone signaled performance for the left hand and a light signaled performance with the right hand (tone-light). According to the notion that the decrement in performance for the tone-tone condition was due to stimulus interference, this decrement should be smaller in the tone-light condition than in the tone-tone condition.

\section{Method}

Independent groups of six subjects were tested with tonetone stimulation (corresponding to Experiment 2) and with tone-light stimulation. Alternate subjects were assigned to the tone-tone and tone-light groups. In the tone-light case, the light was located $10 \mathrm{deg}$ above the right telegraph key, and the tone was presented only to the left ear. Subjects in each group received the identical, skip, and unsynchronized conditions, using the procedures of Experiment 2 . The order in which these conditions appeared was balanced across subjects in each group but remained constant for a given subject across sessions of practice. Each condition was presented as a consecutive set

Table 3

Proportion of Time During Which Stimulus and Response Did Not Correspond for Right Hand in Experiment 2

\begin{tabular}{lcccccccccccc}
\multicolumn{1}{c}{} & \multicolumn{10}{c}{ Day } \\
\cline { 2 - 12 } & & 1 & 2 & 3 & 4 & 5 & 6 & 7 & 8 & 9 & 10 & Mean \\
\hline Skip & .08 & .07 & .07 & .07 & .07 & .07 & .06 & .11 & .07 & .08 & .08 \\
Unsynchronized & .24 & .21 & .23 & .16 & .16 & .17 & .16 & .14 & .16 & .15 & .18 \\
\hline
\end{tabular}


of eight trials, with data scored from the last six trials. Each subject received five sessions on consecutive days. The 12 righthanded subjects were from the same population as in the other experiments. One subject was not able to perform the task and had to be replaced.

\section{Results and Discussion}

Left-hand performance (Table 4) differed among the three conditions $[F(2,20)=20.2, p<.001]$ and improved over the 5 days of practice $[F(4,40)=10.3$, $\mathrm{p}<.001]$. Although the condition and practice variables interacted $[F(8,80)=2.75, p<.01]$, the difference among conditions remained significant on the final day $[F(2,20)=11.66, p<.001]$. The difference among the three conditions was due to a difference between the unsynchronized condition and the compatible conditions (identical and skip) $[\mathrm{F}(1,10)>23, \mathrm{p}<.001$, for both comparisons]. Performance did not differ significantly between the two temporally compatible conditions, identical and skip $[F(1,10)=1.13]$. These results are in agreement with Experiment 2.

Left-hand performance did not differ significantly between the tone-tone and tone-light groups $[F(1,10)=$ 1.28]. Of particular interest for this experiment would be any interaction involving this stimulus modality variable by the performance decrement contrasting the compatible and noncompatible tasks. According to the possibility that this decrement might be due to stimulus perception, the decrement would be smaller for the tone-light group than for the tone-tone group. There was no hint of such an interaction. Indeed, the nonsignificant trend in the data was an interaction of the opposite sense, with a possibly larger decrement for the tone-light group than for the tone-tone group. We conclude that the decrement observed when comparing compatible (identical and skip) conditions with the incompatible condition (unsynchronized) can be attributed to response output limits rather than to perceptual interference. This conclusion is supported by the result (of Experiments 1 and 2) that a decrement is shown in the press fast condition, for which there is no stimulus to guide right-hand performance, and hence no possibility of stimulus interference.

Table 4

Proportion of Time During Which Stimulus and Response Did Not Correspond for Left Hand in Experiment 3

\begin{tabular}{lccccc}
\hline & \multicolumn{5}{c}{ Day of Practice } \\
\cline { 2 - 6 } \multicolumn{1}{c}{ Task } & 1 & 2 & 3 & 4 & 5 \\
\hline \multirow{5}{*}{ Tone/Tone Group } \\
Unsynchronized & .297 & .226 & .193 & .190 & .154 \\
Skip & .176 & .143 & .116 & .128 & .129 \\
Identical & .158 & .118 & .115 & .130 & .135 \\
& \multicolumn{5}{c}{ Tone/Light Group } \\
Unsynchronized & .358 & .343 & .307 & .266 & .246 \\
Skip & .183 & .170 & .172 & .137 & .134 \\
Identical & .149 & .132 & .137 & .123 & .114 \\
\hline
\end{tabular}

\section{EXPERIMENT 4}

The first three experiments showed that two responses that were not temporally compatible (periods were not harmonically related) interfered with each other, but temporally compatible responses did not interfere with each other. However, the conclusion of no interference for compatible responses is limited in that all pairs of responses studied shared a common time of initiation or triggering, even when the periods were different. ${ }^{2}$ Perhaps compatible responses that differ in time of triggering would interfere with each other, contrary to the expectations of the theoretical notions under consideration. Experiment 4 explored that possibility.

\section{Method}

Conditions. The left hand was to attempt the same response in all conditions: key down for $.6 \mathrm{sec}$ (left tone on), key up for $1.8 \mathrm{sec}$ (left tone off), period $2.4 \mathrm{sec}$. A tone in the right ear signaled the right-hand response, which differed among the four conditions. In the "same period, same trigger" condition, the right-hand response was identical to the left-hand response. In the "same period, different trigger, no overlap" condition, the right-hand response was identical to the left-hand response in temporal pattern, but it was displaced in phase such that the two buttonpresses would not overlap in time. The .6 -sec righthand press response was centered in the 1.8-sec interval of no pressing with the left hand, and vice versa. In the "same period, different trigger, overlap" condition, the left- and right-hand press responses were of the same temporal pattern, but the phase relation was such that one hand pressed first, followed after $.3 \mathrm{sec}$ (halfway through the .6-sec press interval) with the start of the .6-sec press of the other hand. For half of the subjects, the right-hand response preceded the left, and this relation was reversed for the remaining subjects. The above three conditions had the same period of repetition $(2.4 \mathrm{sec})$ for the left- and right-hand responses. Therefore, left-hand performance should be equivalent for the three conditions, even though the time of triggering of the press response differed between the left and right hands in two of the conditions.

The fourth condition was "different period, different trigger." For half of the subjects, the duration of the tone-off interval was $1.6 \mathrm{sec}$ (total period $2.2 \mathrm{sec}$ ), and for the remaining subjects, the tone-off interval was $2.0 \mathrm{sec}$ (total period $2.6 \mathrm{sec}$ ). This right-hand task should produce more interference with lefthand responding according to the notion under test.

Design. Each condition was presented as a block of eight trials, with each trial involving a 20-sec period of measured performance, as in the previous experiments. Data were scored from the final six trials. All four conditions were presented to each subject in each of 10 daily sessions, with the order of conditions balanced across subjects but constant across days for any given subject.

Subjects. The eight subjects were from the same population as in the previous experiments. One subject was replaced before the experiment was completed because she showed a proportion of time in error approximately twice that of the next worse subject and did not improve over 5 days of practice.

\section{Results and Discussion}

Left-hand performance differed among the four conditions $[F(3,21)=16.9, p<.001]$ and improved over the 10 days of practice $[F(9,63)=5.5, p<.001]$. There was no significant interaction of practice by 
conditions $[F(27,189)=1.2, p>.2]$. In agreement with Experiment 2, the other 10-day experiment, performance stabilized after 5 days, with no further improvement over the last 5 days $[F(4,28)<1]$. Since we are primarily concerned with stabilized performance after practice, only data from the last 5 days will be considered (Table 5). ${ }^{3}$ Left-hand performance differed among the four conditions averaged over only the last 5 days $[\mathrm{F}(3,21)=12.6, \mathrm{p}<.001]$.

Consistent with expectation (and with the other experiments), left-hand performance was markedly worse in the condition for which the periods of repetition for left- and right-hand responses were not harmonically related (different period, different trigger), compared with the other conditions $[F(1,7)=13.2$, at least, $\mathrm{p}<.005$, for all relevant comparisons].

The other prediction was that left-hand performance would be identical in the three conditions involving the same period for left- and right-hand responses, regardless of whether triggering was simultaneous or staggered. As expected, performance did not differ between the two conditions for which the press responses overlapped in time (same period, same trigger, compared with same period, different trigger, partial overlap) $[F(1,7)<1]$, suggesting that harmonically related responses that do not correspond in triggering interfere with each other no more than do two identical responses. However, performance was better for the same period, different trigger, no overlap condition than for the same period, same trigger, total overlap condition $[F(1,7)=9.3, p<.025]$. Thus, responses differing in triggering can be even easier to perform together than responses that correspond in triggering.

These results are in agreement with the view that nonharmonic responses interfere with each other, but different responses that are harmonically related are no harder to do together than are two identical responses even if the harmonically related responses do not share a common trigger for initiation. However, the results do not correspond to the notion that two responses can be done in parallel without mutual interference if they are temporally compatible. Rather, the results show that temporally compatible responses that do not share a common time of muscular activation interfere with each other less than responses that overlap in time of muscular activation. Looking back at Experiment 1 (Table 1), the reader will note that there was a nonsignif-

Table 5

Proportion of Time During Which Stimulus and Response Did Not Correspond for Left Hand in Experiment 4 on the Last 5 Days of Practice

\begin{tabular}{llll}
\hline Period & Trigger & Overlap & $\begin{array}{r}\text { Propor- } \\
\text { tion }\end{array}$ \\
\hline Same & Same & Total & .112 \\
Same & Different & Partial & .115 \\
Same & Different & None & .081 \\
Different & Different & & .154 \\
\hline
\end{tabular}

icant trend suggesting the possibility that performance was slightly better with the right hand idle than with identical left- and right-hand responses. This trend was more notable at the faster responding rates. This trend also suggests the performance may be degraded by overlap of muscular activity.

\section{GENERAL DISCUSSION}

When the left and right hands pressed telegraph keys, the two responses interfered with each other if their periods were not harmonically related. This interference can be attributed to limitations of response generation rather than to limitations of stimulus perception. By contrast, responses that were temporally compatible in the sense of having harmonically related periods interfered with each other no more than did two identical responses, even when the two responses did not share a common triggering or initiation time. These results support the general notion that responses on different temporal frames can be generated in parallel only at the expense of mutual interference. ${ }^{4}$

Other data consistent with this view have been reported by Kelso, Southard, and Goodman (1979), who employed a target-aiming task in which simultaneous movements were carried out with the right and left hands. According to Fitts' law (Fitts \& Peterson, 1964), movement duration should decrease as this task is made easier by the use of larger targets. Kelso et al. (1979) examined the case in which the right and left hands were to move to targets of different sizes. If Fitts' law were to hold for each hand separately, the two hands would move at different speeds, and the two movements would have different durations. However, the two hands tended to move in the same temporal pattern, with the timing determined by the more difficult of the two targets. ${ }^{5}$ This result suggests that subjects use a single time base to control the two hands, in order to avoid performance degradation that would occur if two independently timed responses were attempted.

Another way of phrasing the notion of a single time base dominating response production would emphasize the power of rhythm to organize and dominate responses. Lashley (1951) noted that "rhythms tend to spread to almost every other concurrent activity. One falls into step with band, tends to breathe, and even to speak in time with the rhythm." The present formulation does not take issue with Lashley's observation, but it does emphasize that a unitary timing system may dominate action even when no strong rhy thmic pattern is present.

The tendency of the nervous system to generate only one temporal pattern at a time can perhaps be attributed to the wealth of interconnections within the system. Although individual neural subsystems might be capable of generating independent rhythmic patterns if the regions were isolated from each other, the entire system may be locked into synchrony to one rhythm by 
the interconnections. Thus, the present findings can be accommodated without assuming that the nervous system has insufficient neural tissue to handle dual timing. Presumably, increasing the size of an interconnected brain that is locked in synchrony in this manner would not expand the range of temporal patterns that can be handled simultaneously.

It should be clear, especially from the last paragraph, that this dual-task limit falls into the category of a structural capacity limit at a fixed locus within the flow of information, rather than a limited capacity that can be allocated to different processing stages (Kahneman, 1973; Moray, 1967). It is not the case that structural and capacity limits are mutually exclusive concepts. The capacity-limit model may turn out to fit data from most stages of processing, including, as Logan (1978) has suggested, all of the stages described by Smith (1968): encoding, comparison, decision, and response selection. However, the often overlooked but logically necessary terminal stage of information processing in which response commands are programmed or organized (Klapp, 1976, 1978) may, as is proposed here, have a limitation concerning multiple responding for which a structural description is appropriate. Previous research may have tended to overlook this aspect of dual task limits, not so much because the limitation is difficult to observe, but because the experimental paradigms have focused on the earlier processes and therefore have not included manipulations of temporal compatibility that would reveal this limitation on doing two things at once.

\section{REFERENCES}

Cralk, K. J. W. Theory of the human operator in control systems II. Man as an element in a control system. British Journal of Psychology, 1948, 38, 142-148.

Deutsch, D. The psychology of music. In E. C. Carterette \& M. P. Friedman (Eds.), Handbook of perception: Perceptual coding (Vol. 8). New York: Academic Press, 1978.

Fitts, P. M., \& Peterson, J. R. Information capacity of discrete motor responses. Journal of Experimental Psychology', $1964,67,103-112$

Greenwald, A. G., \& Shulman, H. G. On doing two things at once: II. Elimination of the psychological refractory period effect. Journal of Experimental Psychology, 1973, 101, 70-76.

Herman, L. M., \& Kantowitz, B. H. The psychological refractory period effect: Only half of the double-stimulation story? Psychological Bulletin, 1970, 73, 74-88.

Johnston, W. A., \& Heinz, S. P. Flexibility and capacity demands of attention. Journal of Experimental Psychology: General, 1978, 107, 420-435.

Johnston, W. A., \& Heinz, S. P. Depth of nontarget processing in an attention task. Journal of Experimental Psychology: Human Perception and Performance, 1979, 5, 168-175.

Kahneman, D. Attention and effort. Englewood Cliffs, N.J: Prentice-Hall, 1973

Kelso, J. A. S., Soutiand, R. L., \& Goodman, D. On the coordination of two-handed movements. Journal of Experimental Psychology: Human Perception and Performance, 1979, 5. 229-238.

KLAPP, S. T. Short-term memory as a response preparation state. Memory \& Cognition, 1976, 4, 721-729
KLAPP, S. T. Reaction time analysis of programmed control. In R. Hutton (Ed.), Exercise and sports sciences reviews. Santa Barbara: Calif: Journal Publishing Affiliates, 1978.

KLAPP, S. T., \& GREim, D. M. Programmed control of aimed movements revisited: The role of target visibility and symmetry. Journal of Experimental Psychology: Human Perception and Performance, in press.

LASHLEY, K. S. The problem of serial order in behavior. In L. P. Jeffress (Ed.), Cerebral mechanisms in behavior: The Hixon symposium. New York: Wiley, 1951

LEwis, J. L. Semantic processing of unattended messages using dichotic listening. Journal of Experimental Psychology, 1970, 85, 225-228.

Logan, G. D. Attention in character-classification tasks: Evidence for the automaticity of component stages. Journal of Experimental Psychology: General, 1978, 107, 32-63.

MORAY, N. Where is capacity limited? A survey and a model. Acla Psychologica, 1967, 27, 84-92.

Schneider, W., \& Shiffrin, R. M. Controlled and automatic human information processing: I. Detection, search, and attention. Psychological Review, 1977, 84, 1-57.

Shiffrin, R. M., \& Schneider, W. Controlled and automatic human information processing: Il. Perceptual learning, automatic attending, and a general theory. Psychological Review, 1977, 84, 127-190.

Smith, E. E. Choice reaction time: An analysis of the major theoretical positions. Psychological Bulletin, 1968, 69, 77-110.

Treisman, A., Squire, R., \& Green, J. Semantic processing in dichotic listening? A replication. Memory \& Cognition, 1974, 2, 641-646.

VINCF, M. A. The intermittency of control of movements and the psychological refractory period. British Journal of Psychology, $1948,38,149-157$

WF.tFord, A. T. Fundamentals of skill. London: Methuen, 1968.

\section{NOTES}

1. Independent groups of four subjects received two slightly different versions of the unsynchronized condition. These subconditions differed in the actual period of the right-hand response, but the right- and left-hand response periods were not harmonically related in either case. These two subconditions seemed to produce about the same pattern of results, and hence the data were combined for presentation.

2. A reviewer of a previous version of this paper (which did not include Experiment 4) pointed this out.

3. The subconditions in the same period, different trigger, overlap and different period, different trigger conditions were equivalent in performance. Therefore, the data were combined across subconditions.

4. Musical polyrhythms (e.g., "3 against 4") can be considered to be an example in support of this conclusion. From one perspective, the left- and right-hand periods between successive beats are not in harmonic relation, and mutual interference should occur. From another perspective, considering the overall periodicity of the repeating pattern, the two responses lie on the same time base and could be produced without interference. It is appealing to assume that, with practice, a musician can leam to regard the response in the latter way rather than in the former way, thereby mastering the task. For a discussion of rhythm in music, see Deutsch (1978, pp. 204-208).

5. Similarly, Klapp and Greim (in press) concluded that two responses differing in duration and direction but both using the right hand cannot both be programmed together. By contrast, two responses of the same duration can be programmed together. 